\title{
CD103 in the development of experimental asthma
}

\author{
Marie-Renee Blanchet", Matthew Gold, Jami Bennett, Kelly McNagny \\ From AllerGEn NCE Inc.'s Fifth Annual Research Conference: Innovation from Cell to Society \\ Québec City, QC, Canada. 7-9 February 2010
}

\section{Objective/purpose}

CD103 (Alpha-E Beta-7 integrin) is expressed on various cell types involved in the development of asthma, including dendritic cells (DCs) and CD4 and CD8 T regulatory cells. This integrin binds E-cadherin on epithelial cells and plays a role in regulating the migration and proliferation of cells. Also, $\mathrm{CD}_{103}{ }^{+}$dendritic cells have been reported to direct the development of naïve $\mathrm{T}$ cells into $\mathrm{T}$ regulatory cells. However, little is known about the exact role of CD103 in the development of asthma. The objective of this project is to investigate the role of this integrin in asthma pathogenesis, in the hope of finding new molecular targets for the treatment of asthma.

\section{Methods}

Asthma was induced in wild type $\mathrm{C} 57 \mathrm{Bl} / 6$ and $\mathrm{Cd} 103^{-/-}$ mice. Briefly, mice were sensitized to Ovalbumin (OVA) through two $100 \mu \mathrm{l}$ intraperitoneal injections of $0.02 \%$ OVA coupled to aluminium hydroxide on days 1 and 8 . Mice were then challenged intranasally with $50 \mu \mathrm{l}$ of $2 \%$ OVA on days $22,23,24,26$ and 28 , and sacrificed on day 29. The assessment of airway inflammation was performed by analysis of the broncho-alveolar lavage (BAL) content as well as the hematopoietic content of collagenase-digested lungs, where the numbers of lymphocytes, macrophages, neutrophils, eosinophils, myeloid dendritic cells, lymphoid dendritic cells, plasmacytoid dendritic cells and $\mathrm{T}$ regulatory cells were determined. Cytokine production in recall to OVA was tested in both lung hematopoietic cells and the draining lymph nodes. Finally, airway hyperresponsiveness was tested via a methacholine challenge, using a flexiVent apparatus.

\section{Findings}

The analysis of airway inflammation revealed that lack of CD103 expression leads to worse asthma compared to wild type mice, as characterized by an increase in total BAL cells, in the percentage and total numbers of eosinophils in the BAL, in cytokine production in recall to OVA and by an increase in airway resistance in response to methacholine. CD103 expression did not seem to affect the accumulation of DCs or T regulatory cells in the lung tissue at baseline and in response to OVA. However, lack of CD103 leads to an expansion of the myeloid dendritic cell population in the lung at baseline, which could account for the exacerbated disease observed in these mice.

\section{Deliverables and relevance}

In light of these results, we believe that a further understanding of CD103 function in the lung could lead to an interesting new molecular pharmacological target. Also, as this molecule is expressed specifically on dendritic cells and $\mathrm{T}$ regulatory cells, this research could lead to new ways of intervening in the process by which these cells drive the asthmatic response rather than a normal, non-allergic response.

Published: 26 November 2010

doi:10.1186/1710-1492-6-S3-P11

Cite this article as: Blanchet et al:: CD103 in the development of experimental asthma. Allergy, Asthma \& Clinical Immunology 2010 6(Suppl 3):P11.

The Biomedical Research Centre, University of British Columbia, Canada 